\title{
Análise dos efeitos da injeção subtenoniana posterior de triancinolona: série de casos
}

\author{
Effects of analysis of posterior subtenon injection of triamcinolone: a case series
}

\author{
Moysés Eduardo Zajdenweber ${ }^{1}$ \\ Remo Turchetti Moraes ${ }^{2}$ \\ Cristina Muccioli ${ }^{3}$
}

${ }^{1}$ Doutor em Medicina pela Universidade Federal de São Paulo - UNIFESP - São Paulo (SP) - Brasil; Colaborador do Setor de Úvea e AIDS do Departamento de Oftalmologia da UNIFESP; Setor de Uveítes do Instituto Brasileiro de Oftalmologia - IBOL - Rio de Janeiro (RJ) - Brasil.

Mestre em Medicina pela Universidade Federal do Rio de Janeiro - UFRJ - Rio de Janeiro (RJ) - Brasil; Setor de Retina do IBOL - Rio de Janeiro (RJ) - Brasil.

${ }^{3}$ Professora Livre Docente da UNIFESP - São Paulo (SP) - Brasil; Chefe do Setor de Úvea e AIDS do Departamento de Oftalmologia da UNIFESP - São Paulo (SP) Brasil.

Endereço para correspondência: Rua Desemb. Renato Tavares, 30/302 - Rio de Janeiro (RJ) CEP 22411-060 E-mail: moysesz@uol.com.br

Recebido para publicação em 04.04.2005

Versão revisada recebida em 05.12 .2005

Aprovação em 09.12.2005

Nota Editorial: Depois de concluída a análise do artigo sob sigilo editorial e com a anuência dos Drs. Áisa Haidar Lani e Procópio Miguel dos Santos sobre a divulgação de seus nomes como revisores, agradecemos sua participação neste processo.

\section{RESUMO}

Objetivo: Avaliar os efeitos da injeção subtenoniana posterior de acetato de triancinolona em pacientes com inflamação intra-ocular. Métodos: Série de casos em que foram avaliados 24 pacientes (26 olhos) submetidos a injeção subtenoniana posterior de acetato de triancinolona para o tratamento de inflamação intra-ocular. Em todos os pacientes foi associado o maleato de timolol $0,5 \%$ por 30 dias. Resultados: Foi observado melhora da acuidade visual em $81 \%$ dos pacientes após injeção única de acetato de triancinolona. Não foi observado aumento significativo da pressão intraocular em nenhum dos olhos tratados. Conclusão: $\mathrm{O}$ acetato de triancinolona sob a forma de injeção subtenoniana posterior mostrou ser uma forma eficaz e segura de tratamento para as inflamações intra-oculares.

Descritores: Corticosteróides; Inflamação; Triancinolona; Pressão intra-ocular; Acuidade visual

\section{INTRODUÇÃO}

Os corticosteróides são drogas freqüentemente utilizadas em oftalmologia, tendo suas principais indicações no tratamento das uveítes de diferentes etiologias. A escolha entre as diversas vias de administração dos corticosteróides vai depender do tipo e localização da uveíte, assim como da intensidade da inflamação e das características clínicas do paciente ${ }^{(1)}$.

$\mathrm{O}$ acetato de triancinolona é um corticosteróide de depósito muito utilizado por via subtenoniana, que atinge concentrações intra-oculares terapêuticas após a sua absorção transescleral ${ }^{(1)}$, ao mesmo tempo que sua pequena absorção sistêmica minimiza os inúmeros efeitos adversos extraoculares. Os efeitos colaterais locais incluem o aumento da pressão intraocular $^{(2-3)}$, catarata $^{(3)}$, oclusões vasculares ${ }^{(4)}$, necrose conjuntival ${ }^{(5)}$, leucoderma adquirido ${ }^{(6)}$, além da penetração acidental do globo ocular.

A injeção periocular de corticosteróides de depósito é utilizada há vários $\operatorname{anos}^{(7)}$, sendo uma opção terapêutica que permite ação contínua da droga evitando, assim, a necessidade de injeções subconjuntivais sucessivas de corticosteróides de curta ação. Além disso, o seu uso local minimiza os inúmeros efeitos adversos extra-oculares da corticoterapia sistêmica.

O presente estudo tem como objetivo avaliar os efeitos da injeção subtenoniana posterior de triancinolona em pacientes com inflamação intra-ocular.

\section{MÉTODOS}

Série de casos onde 24 pacientes (26 olhos) foram examinados e submetidos a injeção de acetato de triancinolona (Kenalog ${ }^{\circledR} 40 \mathrm{mg}$ ) para tratamen- 
to de inflamação intra-ocular no período de março de 2000 a agosto de 2003. Todas as aplicações, injeção de $1 \mathrm{ml}$ (40 mg) de triancinolona utilizando seringa e agulha de insulina, foram feitas pelo mesmo especialista (M.E.Z.) sob anestesia tópica, no quadrante temporal superior. Todos os pacientes utilizaram maleato de timolol 0,5\% tópico durante 30 dias a partir da data da injeção. Nenhum paciente apresentava história pregressa de aumento da pressão intra-ocular assim como nenhum paciente apresentava pressão intra-ocular maior que $16 \mathrm{mmHg}$ no momento da indicação do tratamento.

Foram medidas a acuidade visual (tabela de Snellen) e pressão intra-ocular nos dias 1, 7, 30, 90 e 180 .

Todos os pacientes receberam injeção única de acetato de triancinolona, sendo que dois pacientes se submeteram a injeção, não simultânea, em ambos os olhos.

Quanto ao sexo 8 eram do sexo masculino e 16 do sexo feminino. A idade variou de 18 a 81 anos com média de 25 anos.

\section{RESULTADOS}

Os pacientes apresentavam inflamação intra-ocular diagnosticadas como: vitreíte e edema macular cistóide (9 pacientes), papilite (6 pacientes), coroidite (4 pacientes), uveíte intermediária (3 pacientes), vasculite (1 paciente, 2 olhos) e doença de Behçet (1 paciente, 2 olhos). A pressão intra-ocular pré- injeção média foi de $14 \mathrm{mmHg}$ (variando de 10-16 mmHg) e a pressão intra-ocular pós-injeção média (180 dias) foi de $14 \mathrm{mmHg}$ (variando de 10-16 mmHg) (Quadro 1). Um paciente apresentou pressão intra-ocular de $17 \mathrm{mmHg}$ no $7^{\circ}$ dia pós-injeção.

Quanto à acuidade visual, todos os pacientes apresentavam acuidade visual inicial pior ou igual a 20/40 (Quadro 1).

Avaliou-se os resultados em relação a acuidade visual através da média aritmética simples, onde se dividiu soma das observações pelo número total da amostra.

A acuidade visual melhorou, pelo menos 1 linha, em $81 \%$ dos olhos tratados. Sendo que 20\% melhoraram 1 linha e $58 \%$ melhoraram pelo menos 3 linhas.

Nenhum olho apresentou diminuição da acuidade visual.

Cinco olhos não apresentaram melhora da acuidade visual, sendo que em todos os 5 a acuidade visual inicial era menor ou igual a 20/200 (Quadro 1).

Um paciente apresentou discreta blefaroptose que regrediu espontaneamente. Nenhuma outra complicação foi observada a curto, médio ou longo prazo.

\section{DISCUSSÃO}

O uso do acetato de triancinolona vem apresentando um crescente uso dentro da oftalmologia seja por injeção subtenoniana ou por injeção intravítrea. Porém o seu uso não é

\begin{tabular}{|c|c|c|c|c|c|c|c|c|}
\hline \multicolumn{9}{|c|}{ Quadro 1. Características dos pacientes tratados } \\
\hline Paciente & Idade & Sexo & Diagnóstico & $\begin{array}{l}\text { Olho } \\
\text { tratado }\end{array}$ & $\begin{array}{l}\text { Pio inicial } \\
(\mathrm{mmHg})\end{array}$ & $\begin{array}{l}\text { Pio final } \\
\text { (mmHg) }\end{array}$ & $\begin{array}{c}\text { AV } \\
\text { inicial }\end{array}$ & $\begin{array}{c}\text { AV } \\
\text { final }\end{array}$ \\
\hline 1 & 61 anos & Fem. & Uveíte intermediária & OE & 12 & 13 & $20 / 50$ & $20 / 30$ \\
\hline 2 & 20 anos & Masc. & Coroidite & OD & 10 & 15 & $20 / 200$ & $20 / 40$ \\
\hline 3 & 34 anos & Fem. & Papilite & OD & 12 & 12 & $20 / 40$ & $20 / 20$ \\
\hline 4 & 26 anos & Masc. & Papilite & OD & 12 & 12 & $20 / 40$ & $20 / 30$ \\
\hline 5 & 45 anos & Fem. & Uveíte intermediária & OE & 10 & 11 & $20 / 400$ & $20 / 40$ \\
\hline 6 & 54 anos & Fem. & Papilite & OD & 13 & 12 & $20 / 50$ & $20 / 30$ \\
\hline 7 & 70 anos & Fem. & Vitreíte + edema macular cistóide & OE & 13 & 16 & $20 / 200$ & $20 / 100$ \\
\hline 8 & 46 anos & Masc. & Vitreíte + edema macular cistóide & OE & 14 & 16 & $20 / 100$ & $20 / 40$ \\
\hline 9 & 81 anos & Fem. & Vitreíte + edema macular cistóide & OE & 11 & 13 & $20 / 150$ & $20 / 50$ \\
\hline 10 & 76 anos & Masc. & Vitreíte & OE & 12 & 12 & Vultos & $20 / 100$ \\
\hline 11 & 18 anos & Fem. & Uveíte intermediária & OE & 13 & 12 & $20 / 200$ & $20 / 20$ \\
\hline 12 & 19 anos & Fem. & Behçet & OD & 12 & 14 & $20 / 200$ & $20 / 200$ \\
\hline 12 & 19 anos & Fem & Behçet & OE & 14 & 12 & $20 / 200$ & $20 / 60$ \\
\hline 13 & 50 anos & Masc. & Papilite & OE & 10 & 12 & $20 / 200$ & $20 / 50$ \\
\hline 14 & 20 anos & Fem. & Papilite & OD & 12 & 13 & $20 / 400$ & $20 / 25$ \\
\hline 15 & 74 anos & Masc. & Vitreíte + edema macular cistóide & OE & 13 & 13 & $20 / 200$ & $20 / 30$ \\
\hline 16 & 62 anos & Fem. & Vitreíte + edema macular cistóide & OD & 12 & 12 & $20 / 200$ & $20 / 30$ \\
\hline 17 & 66 anos & Fem. & Vitreíte + edema macular cistóide & OD & 11 & 12 & $20 / 100$ & $20 / 25$ \\
\hline 18 & 60 anos & Fem. & Coroidite & OD & 12 & 12 & $20 / 50$ & $20 / 40$ \\
\hline 19 & 53 anos & Fem. & Edema macular cistóide & OE & 10 & 16 & $<20 / 400$ & $<20 / 400$ \\
\hline 20 & 19 anos & Fem. & Coroidite & OE & 13 & 11 & $<20 / 400$ & $<20 / 400$ \\
\hline 21 & 39 anos & Masc. & Vitreíte & OD & 12 & 12 & Mov. de mão & Mov. de mão \\
\hline 22 & 70 anos & Fem. & Vasculite & OD & 16 & 16 & $20 / 40$ & $20 / 30$ \\
\hline 22 & 70 anos & Fem. & Vasculite & $\mathrm{OE}$ & 10 & 12 & $20 / 100$ & $20 / 40$ \\
\hline 23 & 44 anos & Fem. & Vitreíte & OE & 10 & 11 & $<20 / 400$ & $20 / 80$ \\
\hline 24 & 31 anos & Masc. & Coroidite & OE & 12 & 12 & $<20 / 400$ & $<20 / 400$ \\
\hline
\end{tabular}


isento de riscos, várias complicações podem ocorrer, como perfuração de globo ocular, oclusões vasculares, catarata, blefaroptose, proptose, estrabismo, infecções e quemose ${ }^{(7-10)}$. Dentre todas as possíveis complicações, aquela que deve-se ter um cuidado especial, seja devido a sua frequiência, seja devido a sua gravidade é o aumento da pressão intra-ocular ${ }^{(2)}$.

Neste estudo não foram observadas complicações de acidentes de injeção como: perfurações ou quemose. Também não ocorreram outras complicações como: oclusões vasculares, catarata, proptose, estrabismo ou infecções. Em um caso $(0,2 \%)$ ocorreu blefaroptose, que apesar de ser motivo de queixa do paciente, regrediu espontaneamente.

Finamor et al. ${ }^{(10)}$ e Mueller et al. ${ }^{(2)}$ encontraram em seus estudos uma freqüência de aproximadamente $35 \%$ de aumento significativo da pressão intra-ocular, sendo que no estudo de Finamor $^{(10)}$ este aumento foi mais freqüente em crianças.

No presente estudo não foi observado aumento significativo da pressão intra-ocular, talvez se possa atribuir este dado ao uso profilático de colírio anti-hipertensivo (maleato de timolol 0,5\%).

Deve-se ainda considerar como outra possível causa a faixa etária mais elevada dos pacientes neste trabalho.

O maleato de timolol $0,5 \%$ é um colírio de baixo custo e bastante seguro. Deve-se ter um cuidado especial com indivíduos asmáticos e em algumas cardiopatias, porém em geral é uma medicação bem tolerada, sua posologia é bastante confortável (12/12 horas) e seus efeitos colaterais são pouco freqüientes.

Neste estudo nenhum paciente apresentou efeito adverso ao uso do maleato de timolol $0,5 \%$ e a aderência ao tratamento foi total. Os autores acreditam que o seu uso foi a principal causa pela qual não foi observado aumento significativo da pressão intra-ocular.

Estudos anteriores ${ }^{(8,11)}$ mostram o aparecimento de glaucoma mais tardiamente, até 13 meses após a injeção. Neste estudo o acompanhamento dos pacientes foi de 6 meses, talvez um seguimento maior destes pacientes possa mostrar um resultado diferente em relação ao aumento da pressão intra-ocular no presente estudo.

Em relação a acuidade visual a injeção de acetato de triancinolona mostrou uma boa eficácia, melhora de pelo menos 1 linha em $81 \%$ dos olhos tratados e de 3 ou mais linhas em $58 \%$ dos olhos tratados, eficácia esta semelhante ao estudo de Finamor et al. ${ }^{(10)}$.

\section{CONCLUSÃO}

Apesar de uma amostra pequena este estudo demonstra que o tratamento de inflamações intra-oculares com injeção subtenoniana de acetato de triancinolona (Kenalog ${ }^{\circledR}$ ) se mostrou benéfico para os pacientes.

O uso profilático de maleato de timolol 0,5\% se mostrou seguro e parece exercer uma importante função na prevenção do aumento da pressão intra-ocular.

Estudos clínicos randomizados devem ser realizados para se estabelecer uma rotina quanto ao uso destas medicações.

\section{ABSTRACT}

Purpose: To evaluate the effects of posterior subtenon injection of triamcinolone acetonide in patients with intraocular inflammation. Methods: Case series enrolling 24 patients (26 eyes) submitted to posterior subtenon injection of triamcinolone acetonide for intraocular inflammation treatment. Maleate timolol at $0.5 \%$ was prescribed for all patients for 30 days. Results: Improvement of visual acuity was observed in $81 \%$ of the patients after a single injection of triamcinolone acetonide. Increase in the intraocular pression was not observed in the treated eyes. Conclusion: Posterior subtenon injection of triamcinolone acetonide proved to be a safe and effective way of treating intraocular inflammations.

Keywords: Adrenal cortex hormones; Inflammation; Triamcinolone; Intraocular pressure; Visual acuity

\section{REFERÊNCIAS}

1. Kalina PH, Erie JC, Rosenbaum L. Biochemical quantification of triamcinolone in subconjunctival depots. Arch Ophthalmol. 1995;113(7):867-9.

2. Mueller AJ, Jian G, Banker AS, Rahhal FM, Capparelli E, Freeman WR. The effect of deep posterior subtenon injection of corticosteroids on intraocular pressure. Am J Ophthalmol. 1998;125(2):158-63.

3. Rothova A. Corticosteroids in uveitis. Ophthalmol Clin North Am. 2002;15 (3):389-94. Review.

4. Moshfeghi DM, Lowder CY, Roth DB, Kaiser PK. Retinal and choroidal vascular occlusion after posterior sub-tenon triamcinolone injection. Am J Ophthalmol. 2002;134(1):132-4.

5. Agrawal S, Agrawal J, Agrawal TP. Conjunctival ulceration following triamcinolone injection. Am J Ophthalmol. 2003;136(3):539-40.

6. Gallardo MJ, Johnson DA. Cutaneous hypopigmentation following a posterior sub-tenon triamcinolone injection. Am J Ophthalmol. 2004;137(4):779-80.

7. Sturman RM, Laval J, Sturman MF. Subconjunctival triamcinolone acetonide. Am J Ophthalmol. 1966;61(1):155-66.

8. Helm CJ, Holland GN. The effects of posterior subtenon injection of triamcinolone acetonide in patients with intermediate uveitis. Am J Ophthalmol. 1995; 120(1):55-64.

9. Andrade REA, Muccioli C, Farah ME. Injeção intravítrea de acetato de triancinolona no tratamento da síndrome de Vogt-Koyanagy-Harada. Arq Bras Oftalmol. 2004 2004(67):401-6.

10. Finamor LP, Dimantas MAP, Campos VE, Pra Júnior JA, Muccioli C. Efeitos da injeção subtenoniana posterior de corticóide em pacientes com uveíte. Arq Bras Oftalmol. 2003;66(3):289-91

11. Mills DW, Siebert LF, Climenhaga DB. Depot triamcinolone-induced glaucoma. Can J Ophthalmol. 1986;21(4):150-2. 